\title{
1 Experimental and DEM analyses on 2 wheel-soil interaction
}

3

4 Mingjing Jiang ${ }^{1}$, Yongsheng $\mathrm{Dai}^{2}$, Liang Cui ${ }^{3}$, Banglu $\mathrm{Xi}^{4}$

5

$6{ }^{1}$ Professor, "Distinguished Professor of Tongji University”, Dept. of Geotechnical 7 Engineering and State Key Laboratory Disaster Reduction in Civil Engineering,

8 Tongji University, Shanghai, 200092, China (corresponding author).

9 E-mail: mingjing.jiang@ tongji.edu.cn

10 Fax: 0086-21-65985210; Tel: 0086-21-65980238

$11{ }^{2}$ Former MSc student, Dept. of Geotechnical Engineering, College of Civil 12 Engineering, Tongji University, Shanghai 200092, China.

13 E-mail: dys1988@126.com

$14{ }^{3}$ Lecturer, Dept of Civil and Environmental Engineering, University of Surrey, 15 Guildford, Surrey, GU2 7XH, UK

16 E-mail: 1.cui@surrey.ac.uk

$17{ }^{4}$ PhD student, Dept. of Geotechnical Engineering, College of Civil Engineering, 18 Tongji University, Shanghai 200092, China.

19 E-mail: xibanglu@126.com 
26 Abstract: In this paper, the wheel-soil interaction for a future lunar exploration mission 27 is investigated by physical model tests and numerical simulations. Firstly, a series of 28 physical model tests was conducted using the TJ-1 lunar soil simulant with various 29 driving conditions, wheel configurations and ground void ratios. Then the 30 corresponding numerical simulations were performed in a terrestrial environment 31 using the Distinct Element Method (DEM) with a new contact model for lunar soil, 32 where the rolling resistance and van der Waals force were implemented. In addition, 33 DEM simulations in an extraterrestrial (lunar) environment were performed. The 34 results indicate that tractive efficiency does not depend on wheel rotational velocity, 35 but decreases with increasing extra vertical load on the wheel and ground void ratio. 36 Rover performance improves when wheels are equipped with lugs. The DEM 37 simulations in the terrestrial environment can qualitatively reproduce the soil 38 deformation pattern as observed in the physical model tests. The variations of traction 39 efficiency against the driving condition, wheel configuration and ground void ratio 40 attained in the DEM simulations match the experimental observations qualitatively. 41 Moreover, the wheel track is found to be less evident and the tractive efficiency is 42 higher in the extraterrestrial environment compared to the performance on Earth.

43 Keywords: Wheel-soil interaction system; TJ-1 lunar soil simulant; Contact model; 44 DEM simulation; Tractive efficiency

45 46 47 48 49 50 


\section{Introduction}

China has just accomplished the second phase of the Chang'e Lunar Exploration Program in 2013 with a successful landing of an unmanned lunar rover. However, it has only travelled in a limited area and performed simple missions for safety reasons. In the future exploration, the new lunar rover will be required to travel longer distances and carry more equipment, which is more challenging. Therefore, it is necessary to test the rover locomotion under different driving conditions on Earth prior to its deployment on the lunar surface. Many experimental investigations have been conducted on Earth to guide the rover mechanical design (Apostolopoulos, 2001), structural optimization (Patel et al., 2004), locomotion control (Iagnemma et al., 2001), and path planning and following (Ishigami et al., 2006; 2007; Wong, 2012). Moreover, in order to study the gravity effects, Kobayashi et al. (2010) performed physical tests on an aircraft performing parabolic flight maneuver. But the tests are costly and may not be available for most researchers. Alternatively, the Distinct Element Method (DEM) is more efficient in generating virtual extraterrestrial environment (EX), and has become a popular method in recent studies (Nakashima et al., 2006; Medina and Zeghal, 2006; Hopkins et al., 2008; Li et al., 2010; Jiang et al., 2014). However, conventional contact models for disks or spheres lead to a low internal friction angle of the DEM ground, with mechanical behavior different from the lunar soil ground remarkably. Hence, comprehensive grain shapes (Knuth et al., 2012; Johnson et al., 2015) or contact models (Jiang et al., 2005; Jiang et al., 2013; Jiang et al., 2017b) were used to reproduce the high internal friction angles of the test materials. In addition, the previous research usually focused on the wheel performance and sinkage, little attention was paid on the trace distance, which is also an indicator of the wheel-soil interaction and could be used to predict the slip ratio (Ding et al., 2009)

In this paper, the soil-wheel interaction is investigated using numerical studies in combination with physical model tests. Firstly, a series of soil-wheel tests was 
conducted on the TJ-1 lunar soil simulant with various driving conditions, wheel configurations and ground void ratios. Here, the quantitative relationship between the track length and slip ratio described by Ding et al., (2009) was validated for real-time prediction of slip ratio. The variation of tractive efficiency was investigated as a function of driving condition, ground condition and wheel configuration. Then the corresponding simulations were performed using DEM with the lunar contact model including the rolling resistance and the van der Waals force (Jiang et al., 2013) and then validated by comparing with the experimental results. Finally, DEM simulations of wheel performance in an extraterrestrial (lunar) environment were performed to compare the wheel-soil interactions in the terrestrial environment $(\mathrm{T})$ with those in the extraterrestrial environment. Compared with our previous work (Jiang et al., 2017a), which proposed a design guideline for rover wheel testbeds and presented the comparisons between the experimental and analytical results, this current work focuses on the validation of the DEM simulation and the effects of the gravitational acceleration. In addition, more tests conditions are included in this paper, such as different ground void ratios and wheel forms.

\section{Physical model tests}

\subsection{Introduction of wheel-soil interaction system}

The wheel-soil interaction system consists of a glass-walled soil bin, a test wheel, a carriage, two rails, a motor and four sensors. The soil bin has a dimension of $1500 \mathrm{~mm} \times 900 \mathrm{~mm} \times 900 \mathrm{~mm}$, enabling us to conduct tests using wheels with a certain range of radii and widths. The wheel used in the present study has a radius of $125 \mathrm{~mm}$ and width of $100 \mathrm{~mm}$. The rails are installed to guide the motion of the wheel and ensure the wheel does not deviate from its moving direction. The carriage allows the wheel to move freely in the vertical direction, and also, a normal load can be easily applied on the wheel by adding deadweight on the carriage. Fig. 1 provides the overall 
105 view of wheel-soil interaction system used in the current study. When the wheel

106 advances, only the rotational velocity is controlled, allowing the slip ratio and natural

107 sinkage to occur. During the test, the sinkage is measured via two linear-potentiometer

108 displacement sensors affixed to the carriage. The horizontal velocity of the wheel is

109 recorded to calculate the slip ratio. And the driving torque is measured by a torque

110 sensor installed between the wheel and driving motor.

\section{$111 \quad 2.2$ Experimental methodology}

112 Investigations of soil deformation patterns under rigid wheels or rigid cylinders 113 are very important in studying the wheel-soil interaction (Shikanai et al., 2000;

114 Senatore et al., 2013). Ding et al. (2009) preliminarily studied the soil deformation 115 and proposed a slip ratio estimation method based on lug traces. This method is 116 helpful for real-time slip estimation. However, further studies are essential to examine 117 the applicability of such method as the rover may experience a variety of driving 118 conditions or traverse challenging terrains in planetary explorations. Therefore, in the 119 current study, a number of driving conditions (including various rotational velocities $120 \quad w$ and vertical loads $W$ ) and wheel forms were designed. Meanwhile, various ground 121 conditions with different void ratios $e$ were prepared. Therefore, ten representative 122 operating conditions were planned as tabulated in Table 1. Note that test No's 1-5 with 123 various rotational velocities $w$ and vertical loads $W$ have been presented in the 124 previous research (Jiang et al., 2017a). For each test condition, at least two tests were 125 conducted as a repeatability check. In all experiments, the wheel drawbar pull was $126 \quad 32.5 \mathrm{~N}$.

\section{$127 \quad 2.3$ Experimental material}

128 Samples of lunar soil are very scarce on Earth due to the difficulty in collecting 129 samples from the Moon. Therefore, the TJ-1 lunar soil simulant, which was recently 130 developed by Jiang et al. (2012a), was used to generate the test ground in our tests. 
131 The lunar soil simulant has similar chemical composition, mineralogy, shape and

132 gradation of grains to lunar regolith. The raw materials, red volcanic ash deposits

133 collected from Jingyu County in Jilin Province of China, were dried, crushed, and

134 sieved to obtain materials covering the grain size distribution shown in Fig. 2. The

135 TJ-1 lunar soil simulant demonstrates certain similarities to the lunar regolith in terms

136 of physical and mechanical properties. Compared with other simulants introduced in

137 the literature, TJ-1 has an internal frictional angle as high as $47.4^{\circ}$ and a relatively low

138 cohesion $0.86 \mathrm{kPa}$ at $e=1.0$. TJ-1 lunar soil simulant is an ideal substitute of lunar soil

139 located at $0-30 \mathrm{~cm}$ below lunar surface in the mare region, thus it is an appropriate

140 material for performing the soil-wheel interaction tests.

\section{$141 \quad 2.4$ Experimental results}

\section{$142 \quad 2.4 .1$ Soil deformation and slip ratio}

143 When a rover moves on a terrain, the soil beneath the wheel is compressed and 144 traces are left as a result. This is a common phenomenon in most experiments. Fig. 3 145 provides the left traces in our tests with different lug configurations. As shown in Figs.

146 3(b)-(d), the traces left by the lugs can be easily recognized. For the smooth wheel,

147 there is no lug traces, however, there are intensive vertical stripes caused by wheel 148 slippage as illustrated in Fig. 3(a).

149 Fig. 3 also shows that the distance $L$ between the track prints of two adjacent 150 lugs are virtually equal under similar driving conditions when the wheel is equipped 151 with lugs. Ding et al. (2009) proposed that there is a relationship between the distance

$152 L$ and slip ratio $i$. The slip ratio is an important variable for wheel-soil interaction,

153 which is defined as follows:

$$
i=1-v_{x} / R w
$$

155 where $v_{x}$ is the horizontal velocity of wheel. $R$ equals the wheel radius $r$ for the 156 smooth wheel or the sum of the wheel radius $r$ and the lug height $h$ for the wheels 
157 with lugs. It is obvious that the lugs are very effective in reducing the slip ratio,

158 namely, reducing the risk of spinning, as shown in Fig. 3.

159 When $i$ lies between 0 and 1, the relationship between the distance $L$ and slip

160 ratio $i$ proposed by Ding et al. (2009) can be expressed as:

161

$$
L=(1-i) r_{s} \gamma=(1-i)(r+h / 2) \gamma
$$

162 where $r_{s}$ is the shearing radius of the wheel, $r$ is the wheel radius, $h$ is the lug height

163 and $\gamma$ is the spacing angle of wheel lugs.

164 Fig. 4 provides the comparison between experimental and theorectical results of

165 the trace distance $L$. It shows that although the wheel drives on different terrains or

166 with different wheel forms, the maximum relative error between the experimental and

167 theorectical results is only $6.46 \%$, which is acceptable in engineering applications.

168 Thus it can be reasonablely inferred that this relatinoship between $L$ and $i$ is

169 independent of the driving conditions ( $w$ or $W$ ), and packing states of soil ground.

170

171

172

173

174

175

176

177

178

179

180

181

182

183

\subsubsection{Tractive efficiency}

The wheel reaches a steady moving state after running for several seconds when the drawbar pull force (the difference between the gross traction generated by the driving torque and the motion resistance) is balanced by the towed load. Fig. 5 presents typical curves of the driving torque evolution with time for test No .1. The data acquisition frequency is $40 \mathrm{~Hz}$ and hundreds of raw data can be obtained. The measured data fluctuates periodically in association with the wheel lugs entering and leaving the soil, therefore they are smoothed to show the evolution trend. Fig. 5 shows that the driving torque increases to a peak value rapidly at first and then mobilizes at a stable value. The steady data can be used to calculate the mean values of driving torque in following analysis. In addition, the two mean values of the two repeated experiments are almost the same despite the data fluctuations, proving the repeatability and consistency of the experiments.

The tractive efficiency $(\eta)$ (Code, 2003) is adopted for evaluation of wheel 
mobility and can be estimated as follows:

$$
\eta=\frac{F_{D P} \cdot v_{x}}{T \cdot w}
$$

where $F_{D P}$ is the drawbar pull force, $T$ is the driving torque. Combined with Equation (2), the expression can be rewritten as follows:

$$
\eta=\frac{F_{D P}}{T} \cdot(1-i) \cdot R
$$

Fig. 6 provides the tractive efficiencies for different rotational velocities $w$, vertical loads $W$, ground void ratios $e$ and wheel forms. We can find that: (1) the tractive efficiency shows no apparent difference at different rotational velocities (Tests No. 1, 2 and 3) with the same identical drawbar pull force; (2) the tractive efficiency decays with increasing vertical load applied to the wheel (Tests No. 2, 4 and 5). This is because a larger drawbar pull force is required to drag the increasing weight of a rover (Sutoh et al., 2012), but the drawbar pull force is prescribed to a constant value in the current study and a higher slip ratio occurs as a result of the increasing weight; (3) as expected, the wheel shows better performance when travelling on a denser ground, i.e. lower void ratio (Tests 6, 2 and 7); (4) the smooth wheel experiences high slippage and is easy to get stuck, which exhibits a low tractive efficiency. This deficiency can be significantly improved by attaching lugs. With the given lug height, a minimum number of lug is required to form continuous shearing soil loop (Ding et al., 2009), namely 10mm/20 (Test No. 2) and 20mm/10 (Test No. 8) in the current study. Fig. 6 shows that the contributions of lugs are almost the same in such two conditions by increasing the tractive efficiency about $310 \%$ from the condition with no lugs (Test No. 10). However, the contribution of adding more lugs (in case of $20 \mathrm{~mm} / 20$, Test No. 9) is very limited, which only increases the tractive efficiency by $19.4 \%$ compared with the case of $20 \mathrm{~mm} / 10$ (Test No. 8 ). 


\section{DEM simulations}

\subsection{Contact model for lunar soil}

The contact model used in the conventional DEM only considers shear force and repulsive normal force at the contacts (Cundall and Strack, 1979). In view of this limit, efforts have been made to include other features of particle interaction, such as interparticle rolling resistance (Iwashita and Oda, 1998; Tordesillas and Walsh, 2002; Jiang et al., 2005) and cohesion (Luding, 2008) arising from different mechanism such as chemical bonds (Potyondy and Cundall, 2004; Jiang et al., 2007; Jiang et al., 2012b;2012c; Obermayr et al., 2013), liquid bridge (Jiang et al., 2004), and molecular forces (Modenese et al., 2012). As described in previous studies (Modenese et al., 2012; Jiang et al., 2013; Kulchitsky et al., 2016), van der Waals forces have remarkable contribution to the inter-particle adhesion of lunar soil due to the ultra-high vacuum condition in the lunar environment. Therefore, a contact model proposed by Jiang et al. (2013), which fuses the van der Waals forces and the inter-particle rolling resistance together, was used here to accurately model the lunar soil. This contact model, which has been proved to be able to capture the mechanical properties of the lunar soil reasonably well, has not been fully considered in other DEM simulation. Different from the previous work on the model development (Jiang et al., 2013), the current study focuses on the application of the lunar contact model in wheel-soil interaction simulations. Therefore, the model is only briefly described here for completeness. A detailed description of the model can be found in the paper (Jiang et al., 2013).

The two-dimensional contact model of two discs contacting over a rectangular area includes three components in the normal, tangential and rolling directions. Van der Waals forces are introduced in the normal direction and rolling resistance is added into the rolling direction. The mechanical components of the contact model are presented in Fig. 7. The normal contact model controls the normal interaction, with a 
235 spring reflecting the elastic behavior of the contact, a dashpot allowing viscous energy

236 dissipation, an attractor named WF attractor representing the van der Waals force 237 (WF is short for van der Waals force hereafter), and a divider indicating that no 238 tension is transmitted when two grains are separated. The tangential and rolling 239 contact models are similar to the normal model in principle, both composed of springs 240 for elastic behavior and dashpots for viscous contact damping behavior. In the 241 tangential contact model, a load slider and a WF slider are connected in parallel to 242 consider both the load and van der Waals force contributions to the sliding resistance. 243 In the rolling contact model, a load roller and a WF roller are set in parallel, 244 indicating that the moment transmitted between two grains must overcome the 245 resistance produced by both the load and van der Waals force before unconstrained 246 rotation can occur.

\subsection{Mechanical property of the DEM sample}

\subsubsection{DEM parameters}

The mechanical behaviors were investigated here using two sets of biaxial compression test on specimens with an initial void ratio of 0.20 , one considered the van der Waals force representing the extraterrestrial environment, while the other did

252 not consider such force representing the terrestrial environment, where the WF is 253 negligible. The model parameters adopted in the current study are listed in Table 2 254 and the particle size distribution is provided in Fig. 8, along with the upper and lower 255 bounds of gradation curve of lunar regolith collected in the missions of Apollo11, 12 256 and 14 (Mitchell et al., 1972; Carrier et al., 1991; Carrier, 2003; Chang and Hicher, 257 2009). It is obvious that the employed curve is quite different from that used in 258 experimental investigation due to the constraint of computational costs. Based on 259 previous research (Wang, 2012), nearly 100,000 particles are required to simulate the 260 biaxial test if using the actual particle size distribution of lunar regolith. If 261 reproducing the soil ground for simulating the wheel-soil interaction, it will require 
262 more than one million particles in a simulation, which is unaffordable. Nevertheless,

263 the average particle diameter used in the current study is restricted to the upper bound 264 value of real lunar regolith in order to attain reasonable van der Waals force.

\section{3.2.2 DEM specimen}

266 The DEM specimens used for the biaxial compression test were generated using 267 the multilayer under-compaction method (UCM) proposed by Jiang et al. (2003). 268 Eight layers of particles were generated in a sequential way, with each layer 269 containing 750 particles and randomly deposited into a rectangular container with the 270 height to width ratio of 2:1. To achieve the target planar void ratio of 0.20 , the 271 accumulated layers of particles were compacted to an intermediate void ratio which is 272 slightly higher than the target void ratio when each new layer was added. According 273 to the under-compaction criterion proposed by Jiang et al. (2003), the intermediate 274 void ratios for the accumulated layers were; $e_{p(1)}=0.218, e_{p(1+2)}=0.217, e_{p(1+2+3)}=0.225$, $e_{p(1+2+3+4)}=0.214, e_{p(1+2+3+4+5)}=0.211, e_{p(1+2+3+4+5+6)}=0.210, e_{p(1+2+3+4+5+6+7)}=0.205$ and $276 e_{p(1+2+3+4+5+6+7+8)}=0.20$. The DEM specimen was constrained by four frictionless wall 277 boundaries. Following the one-dimensional compression, the specimen was 278 isotropically compressed to different confining pressures (e.g. $25 \mathrm{kPa}, 50 \mathrm{kPa}$ and $279100 \mathrm{kPa}$ ) which are the same as the indoor triaxial compression tests on the dense 280 samples of TJ-1 lunar soil simulant. Then, the top and bottom walls were moved 281 toward each other at a strain rate of $5 \% / \mathrm{min}$ to axially load the specimen in a 282 quasi-static manner, leading to a higher vertical stress $\sigma_{1}$. The two opposite lateral 283 walls were servo-controlled to maintain a constant confining pressure $\sigma_{2}$ applied on 284 the specimen.

$285 \quad 3.2 .3$ Biaxial compression test results

286 Fig. 9 provides the evolutions of the deviator stress and the volumetric strain in 287 two environments. It shows that shear dilation accompanied with strain softening 288 occurs under both conditions. However, the particles are easy to form clusters due to 
289 the effect of van der Waals force in the lunar environment, thus leading to a more

290 pronounced dilation. Besides, the existence of van der Waals force will further

291 enhance the tangential and rolling strengths at particle contacts, therefore a higher

292 peak deviator stress is also observed as a result. The internal friction angle under the

293 terrestrial environment can be attained as $44.8^{\circ}$ while it increases to $45.8^{\circ}$ as a result

294 of the presence of van der Waals force, meanwhile, the material shows a small

295 cohesion of $1.12 \mathrm{kPa}$ in the extraterrestrial environment. Compared with the internal

296 friction angle of the TJ-1 lunar soil simulant, the void ratio of 0.20 is reasonable for

297 the latter wheel-soil simulation.

$298 \quad 3.3$ Viscous damping

299 Note that the viscous damping should be activated in dynamic simulation process 300 for energy dissipation, such as in the case of wheel-soil interaction. Moreover, the 301 numerical time will not coincide with the physical time if an inappropriate damping 302 value is used. In our study, the damping value was determined by simulating the angle 303 of repose in comparison with the results of the indoor tests (Jiang et al., 2017b). The 304 2D disk simulation is similar to plane strain tests. In soil mechanics, the internal 305 friction angle obtained from plane strain compression test is nearly 1.2 times larger 306 than that obtained from triaxial compression test, which has been directly 307 confirmed by theory, supported by experiment, that 2D and 3D repose angles are 308 quite different (Albert et al. 1997). Nevertheless, 2D DEM simulations are always 309 conducted to capture the strength feature of soils regardless of such differences 310 with carefully selected parameter in geotechnical engineering, such as the internal 311 friction angle, cohesion, as well as the angle of repose for simplicity. This can 312 ensure that the following 2D simulations of soil-wheel interaction with the same 313 parameters can provide reasonable results. In addition, the damping coefficient 314 mainly influences the rate-dependency behavior of soils, which shows limited 315 effect on the soil internal friction (Lade et al., 1997). Thus, any approximate value 
316 of soil internal friction could be used to calibrate their damping coefficient.

317 Consequently, the damping coefficient adopted here was determined by matching 318 the angles of repose obtained in 2D DEM simulations with 3D indoor tests for 319 convenience.

320 Firstly, several indoor experimental soil pouring tests were conducted to examine 321 the angle of repose and the results are provided in Fig. 10. It shows that the angle of 322 repose mainly varies between $30^{\circ}$ and $33^{\circ}$. In the DEM simulation, an assembly of 323 granular particles drops freely down under $1 \mathrm{~g}$ to the ground. Different viscous 324 damping values were used in the trial simulations, from 0.05 to 0.5 and the simulation 325 results were drawn in Fig. 11. It is shown that when the normal and shear viscous 326 damping values are set to be 0.4 , the simulated angle is $31.3^{\circ}$, which is very close to 327 the experimental results. Considering that the wheel-soil simulations were further 328 extended to the extraterrestrial environment, the simulations of repose angle test were 329 re-conducted under different gravitational conditions, from $1 / 6 \mathrm{~g}$ to $6 \mathrm{~g}$ while the 330 damping values were fixed at 0.4. The results were also plotted in Fig. 11. We can 331 find that the angle of repose fluctuates slightly with gravity, which is negligible. This 332 phenomenon is similar to the tests results conducted in an airplane (Kobayashi et al., 333 2009). Therefore, the viscous damping values are set to be 0.4 in the latter 334 simulations.

\subsection{DEM model of wheel-soil interaction}

336 3.4.1 Soil ground

337 In order to establish a wheel-soil interaction model to reproduce the indoor 338 experiment, the model dimension should be scaled down with a proper scaling factor. 339 Otherwise, millions of particles are required to generate the test ground if the model 340 in the simulation is at the same size as the prototype. Referring to the previous 341 research (Nakashima et al., 2010), where acceptable results were attained with the 342 ratios of the wheel diameter to the average particle size ranging from 37.5 to 62.5 . In 
343 this study, the ratio was set to the upper bound at 62.5. Given the average particle

344 diameter of $1 \mathrm{~mm}$, the diameter of the wheel used in simulations can be determined as

$34562.5 \mathrm{~mm}$. Considering the wheel diameter of $250 \mathrm{~mm}$ used in experiments, the scaling

346 factor is 4.0. However, it should be noted that less particles will interact with the

347 wheel elements in the model than those in the prototype as the soil particles are not

348 scaled in the same manner. As a result, the output data may fluctuate intensely.

349 Following the determination of the scaling factor, the multilayer

350 under-compaction method (UCM) was again employed here to generate a

351 homogeneity ground sample before consolidation under gravity. A total of eight layers

352 of particles were generated in a sequential way, with each layer containing about 6875

353 particles (given a total number of 55,000 particles) and randomly deposited into a

354 rectangular container to form the granular ground. To achieve the same target planar

355 void ratio of 0.20 as that of the specimen for element tests, the intermediate void

356 ratios for the accumulated layers were the same as those in Subsection 3.2. During the

357 generation process, the wall-particle friction was set to zero in order to improve the

358 homogeneity, while the inter-particle frictional coefficient was set to be 0.2 in order to

359 produce a dense packing of particles. After that, a $4 \mathrm{~g}$ gravitational acceleration was

360 applied in the test ground to achieve the same gravity-induced stress field, which is

361 inspired by the principle of centrifuge modeling that has been widely used in

362 geotechnical engineering.

363 3.4.2 Wheel and lugs

364 The wheel is represented by a large disk with a radius of $31.25 \mathrm{~mm}$. The lug is 365 approximated by 20 disks with radii of $0.125 \mathrm{~mm}$ clumped together with overlaps 366 equal to the radius, thus giving a height of $2.5 \mathrm{~mm}(1 / 4$ of the lug height of $10 \mathrm{~mm}$ in 367 prototype) and width of $0.25 \mathrm{~mm}$. The height to width ratio of lug is 10:1, same with 368 the one used in experimental tests. When simulating different wheel forms, the lug 369 number and height were modified and it was easily accomplished in the DEM studies. 
370 The configuration of the DEM wheel-soil interaction test is provided in Fig. 12. Table

3713 summarizes the parameters of the wheel elements, where larger normal/shear

372 stiffness was used in order to approximate a rigid wheel. In the simulations, the wheel

373 was servo-controlled in such a way: in the vertical direction, the position of the wheel

374 was adjusted so that the vertical load and the weight of the wheel was balanced by the

375 vertical wheel-soil interaction force; in the horizontal direction, the input towed load

376 was balanced by the resultant horizontal wheel-soil interaction force.

\section{$377 \quad 3.4 .3$ Simulation procedure}

378 In addition to the one-to-one correspondence of physical model tests and 379 numerical simulations, another three simulations, numbered 11, 12 and 13, were 380 added. Compared with test No. 2, the above mentioned three tests are different in 381 rotational velocity $(0.818 \mathrm{rad} / \mathrm{s})$, vertical load $(245 \mathrm{~N})$ and ground void ratio $(0.24)$, 382 respectively. 13 simulations were performed in both terrestrial and extraterrestrial 383 environments, yielding 26 simulations in total. Note that the planar void ratios 384 corresponding to the true void ratios of $0.85,1.0$, and 1.05 are $0.18,0.20$, and 0.22 , 385 respectively. These values were attained based on comparable soil strength.

\subsection{DEM simulation results}

\subsubsection{Soil deformations}

The soil deformations beneath the wheel and the trafficability under different 389 driving conditions have been experimentally investigated in the terrestrial 390 environment as detailed above. Herein the corresponding simulations were performed 391 using the DEM model described above and the studies were further extended to the 392 extraterrestrial environment. The wheel used in experiment was $100 \mathrm{~mm}$ thick while 393 the DEM simulation is two-dimensional, which means the width of wheel is unit one, 394 i.e., $1 \mathrm{~m}$ in the model, 10 times of that in the experiment. Moreover, the size of the 395 wheel was scaled down by a factor of 4 and the gravity was increased by 4 , giving the 
same stress level. Therefore, the input towed load and vertical load listed in Table 1

397 should be scaled by a factor of $4 / 10$, and the torque attained from the simulations

398 should be scaled up by $4 \times 4 / 10$ in order to maintain the same condition as in the 399 experiments.

400 Fig. 13 illustrates the soil deformation beneath the wheel in different 401 environments. The test parameters are the same with those in the indoor test No. 2. 402 The experimental result has been shown in Fig. 3(b), where clear tracks could be 403 observed when the wheel passed by. Similarly, the DEM simulation result in the 404 terrestrial environment (Fig. 13(a)) demonstrates the same tendency, where clear and 405 wavy tracks also formed on the ground surface. But further observation shows that the 406 equally spaced tracks resulted from lug digging is not that obvious in our simulation, 407 which can be explained by the smaller ratio of lug size to soil particle diameter. 408 Nevertheless, we can still draw the conclusion that the employed DEM model can 409 qualitatively describe the formation of tracks. Then the simulation was extended to 410 the extraterrestrial environment as illustrated in Fig. 13 (b). It shows that the tracks 411 are less obvious as the weight and the input towed load were both reduced to $1 / 6$ of 412 those in terrestrial environment.

\subsubsection{Gross traction and motion resistance analyses}

414 In this chapter, we mainly analyze how the gross traction and motion resistance 415 vary. Fig. 14 shows the comparison on gross traction (GT) and motion resistance (MR) 416 in the terrestrial environment (T) as well as those in the extraterrestrial environment 417 (EX) when applying a constant towed load. The gross traction is the summation of all 418 the forward contact forces between soil particles and the wheel, and the motion 419 resistance is the summation of all the backward contact forces between soil particles 420 and the wheel. The difference between the GT and MR is the input towed load (81.25 $421 \mathrm{~N}$ in the terrestrial environment and $13.54 \mathrm{~N}$ in the extraterrestrial environment). The 422 GT and MR vary following the same trend with different driving conditions, ground 
423 void ratios and wheel forms as shown in the figure. Fig. 14(a) shows that there is no

424 clear difference in GT and MR when changing the rotational velocity. Therefore, the 425 rover can move at a relatively fast rotational velocity under the premise of safety 426 assurance. Fig. 14(b) shows that the motion resistance and gross traction increase with 427 the vertical load. This is because that higher vertical load produced a larger sinkage, 428 which in turn increases motion resistance with a potential of causing the wheel to 429 become stuck. Similarly, the wheel will experience larger motion resistance and gross 430 traction when moving in a loose ground due to larger sinkage, as shown in Fig.14(c). 431 Fig. 14(d) shows that the motion resistance and gross traction increase as the lug 432 height changes from 0 to $20 \mathrm{~mm}$. Similar to the experimental results, there is an 433 optimal lug height to obtain the best traction of a wheel. Besides, in terms of 434 generating more gross traction, wheels with 10-20 mm-lugs may show better 435 performance than wheels with $20-10 \mathrm{~mm}$-lugs would. The results obtained in the 436 extraterrestrial environment follow similar trend but have much smaller values as a 437 result of reduced weight and towed load.

\subsubsection{DEM validation of tractive efficiency}

To quantitatively estimate the tractive efficiency in the DEM simulations, the slip ratio is recorded using Equation (1) together with Equation (5):

$$
v_{x}=\frac{\Delta x}{\Delta t}
$$

where $v_{x}$ is the horizontal displacement of the wheel taking place within a time interval $\Delta t$.

Fig. 15 presents the simulation results of slip ratio under different environments for test No. 2. Fig. 15 shows that the input rotational velocity is stable while the slip ratio shows a moderate fluctuation around an average value, which was used in the latter calculation of tractive efficiency.

Meanwhile, the driving torque is also recorded and calculated using Equation (6): 


$$
T=\sum^{m}\left(f_{x} \cdot l_{x}+f_{y} \cdot l_{y}\right)
$$

451 where the summation is over the $m$ particle contacts with the wheel, $f_{x}$ and $f_{y}$ are the 452 horizontal and vertical components of contact force, respectively, $l_{x}$ and $l_{y}$ are the 453 distance from the wheel center to the application lines of $f_{x}$ and $f_{y}$.

454 Fig. 16 provides the evolution of driving torque under different environments for 455 test No. 2. Fig. 16 shows that the raw data are rather noisy as a result of lugs and are 456 smoothed by means of moving average. In contrast to the experimental results as 457 shown in Fig. 5, the driving torque in DEM simulation increases to a peak value as 458 soon as the simulation begins and then fluctuates around a steady value, with no clear 459 increasing trend at the beginning (observed in physical model tests). The main reason 460 for the difference may be that the prescribed velocity of wheel can be imposed 461 instantaneously in DEM simulation, while it required some time to accelerate the 462 wheel from still to the given rotational velocity in physical model test. Anyway, only 463 the steady values are required in our post process, thus it is not problematic with a 464 slight difference in the initial stage.

465 After determining the slip ratio and driving torque in every simulation, the 466 tractive efficiency can then be calculated using Equation (4). Fig. 17 presents the 467 simulation results of tractive efficiency obtained in both terrestrial and extraterrestrial 468 environments under different driving conditions, ground void ratios and wheel forms. 469 For comparison, the experimental results are also provided. Fig. 17 shows that the 470 simulation results in the terrestrial environment are smaller than those obtained from 471 the physical model tests, but can still qualitatively capture the evolution trend. Such 472 difference is mainly resulted from the larger slip ratio obtained in DEM simulation. 473 Moreover, the resulted tractive efficiency obtained in the extraterrestrial environment 474 shows a higher value although the vertical load and the towed load both reduce. This 475 may be because the van der Waals forces can increase the soil cohesion and therefore 476 reduce the possibility of wheel slippage. 


\section{Discussions}

478

479

It is obvious that a two-dimensional simulation cannot accurately represent a three-dimensional deposition of a granular material that consists of spherical particles. 2D particles cannot move in the out-of-plane direction as in 3D simulations, which results that 2D DEM cannot capture the full deformation behavior of soils. In addition, the uses of spherical particles and artificial constraint on particle rotations also limit the accuracy in our simulations. However, the mechanical behaviors of the $2 \mathrm{D}$ and $3 \mathrm{D}$ particles contact are similar to a certain extent with carefully selected parameter, which results in that 2D DEM can capture the strength features of soils. Therefore, 2D simulation results could still provide qualitative illustration of the effects of the lunar environment conditions as stated above. Moreover, the huge number of particles required for a 3D simulation is unaffordable for the current capacity of PCs. Therefore, $2 \mathrm{D}$ simulations are effective ways to reduce the computational costs. It can be seen in the previous figures that the slip ratios in the DEM simulations show higher values than those in the indoor experiments. Such phenomenon may be a result of two reasons: firstly, the out-of-plane constraint necessary to enforce a state of plane strain is not present in the 3D problems. Secondly, the rolling resistance that have been implemented to the contacts can only enhance the strength of soils, while it is still difficult to capture the full characteristic of flowability of a real material, where particles are strongly interlocked due to irregular shapes. However, the 2D DEM simulation model can still be used to predict the evolution trend based on the comparison with indoor physical model tests. Therefore, the slip ratio obtained in the extraterrestrial environment should also be reasonable.

Interestingly, the results of driving torque based on the current DEM model shows a good agreement with indoor physical model tests despite 2D simulations, which can also be explained from two aspects. Firstly, the extra out-of-plane constraint leads to increasing gross traction. Secondly, the higher flowability due to the spherical particle reduced the gross traction. Such two effects may be canceled out 
in our DEM simulations.

506

507

508

509

510

511

512

513

514

515

516

517

518

\section{Conclusions}

In this paper, a series of physical model tests using a new wheel-soil interaction system were conducted. Then the corresponding DEM simulations using a novel contact model considering rolling resistance and van der Waals force were performed. The DEM simulations in the terrestrial environment were validated by comparing with the experimental results. At last, the DEM simulations were further extended to the extraterrestrial environment.

The main conclusions can be drawn as follows:

(1) The aforementioned quantitative relation between track length and slip ratio is validated in both the work by Ding et al. (2009) and this current study, despite of different packing states and mechanical properties of soils. Moreover, this relation is also proved to be accurate when changing the driving condition and wheel forms in the current study, suggesting that this is a common relation in wheel-soil interaction which is helpful for real-time estimation of slip ratio by measuring the track length.

(2) The tractive efficiency shows no clear difference at various rotational velocities, but decreases with increasing extra load and void ratio. In addition, the rover shows better performance when equipping with lugs. Lug configuration of 20-10 mm can improve the wheel performance slightly more than $10-20 \mathrm{~mm}$, but the difference is not very evident. However, increasing the lug number from 10 to 20 will further improve wheel performance but to a smaller extent compared with the improvement with increasing lug number from 0 to 10 .

(3) Compared with the experimental results of the angle of repose performed with TJ-1 lunar soil simulant, an appropriate viscous damping value was determined as 0.4 to simulate the dynamic problem. Moreover, the angle of repose seems to be unaffected by different gravity fields.

(4) The exploited model can qualitatively reproduce the pattern of track formed 
532 in experiments. The track is less evident when performed in the extraterrestrial 533 environment.

534 (5) When generating the same net traction, there is no clear difference in both 535 gross traction and motion resistance when changing rotational velocity. However, the 536 gross traction and motion resistance increase evidently with the vertical load, which 537 may cause the wheel to become stuck as a result of excessively large sinkage. The 538 gross traction and motion resistance also increase with ground void ratio thus it is 539 more difficult to traverse over loose terrain. In addition, the motion resistance as well 540 as gross traction increased as the lug height changes from 0 to $20 \mathrm{~mm}$. Those 541 conclusions are similar in the extraterrestrial environment but to a smaller extent.

542 (6) The developed DEM model could qualitatively predict the evolution of 543 traction efficiency and the difference mainly comes from the overestimated slip ratio 544 in the DEM simulations. The tractive efficiency shows a higher value in the 545 extraterrestrial environment with the same test parameters.

\section{Acknowledgements}

548 The work reported in this paper was funded by National Natural Science Foundation 549 of China with grant number 51639008 and 51579178. We would also like to thank $\mathrm{Mr}$ 550 Chang Fu from Tongji University, China, for his contribution on experiment. All the 551 supports are greatly appreciated

\section{References}

553 Albert, R., Albert, I., Hornbaker, D., Schiffer, P., Barabási, A., 1997. Maximum angle 554 of stability in wet and dry spherical granular media. Physical Review E 56(6), $555 \quad 6271-6274$.

556 Apostolopoulos, D.S., 2001. Analytical configuration of wheeled robotic locomotion.

557 In: The Robotics Institute of Carnegie Mellon University Technical Report 
CMU-RI-TR-01-08.

559 Carrier, W.D., 2003. Particle size distribution of lunar soil. Journal of Geotechnical $560 \quad$ and Geoenvironmental Engineering 129(10), 956-959.

561 Carrier, W.D., Olhoeft, G.R., Mendell, W., 1991. Physical properties of the lunar 562 surface. Lunar sourcebook, 475-594.

563 Chang, C.S., Hicher, P.Y., 2009. Model for granular materials with surface energy 564 forces. Journal of Aerospace Engineering 22(1), 43-52.

565 Code, O.E.C.D., 2003. General Terminology for Traction of Agricultural Traction and 566 Transport Devices and Vehicles. ANSI/ASABE S, 296, 1-5.

567 Cundall, P.A., Strack, O.D., 1979. A discrete numerical model for granular assemblies. 568 Geotechnique 29(1), 47-65.

569 Ding, L., Gao, H., Deng, Z., Yoshida, K., Nagatani, K., 2009. Slip ratio for lugged 570 wheel of planetary rover in deformable soil: definition and estimation. In: The 571 International Conference on Intelligent Robots and Systems, 3343-3348.

572 Hopkins, M. A., Johnson, J. B., Sullivan, R., 2008. Discrete element modeling of a 573 rover wheel in granular material under the influence of Earth, Mars, and Lunar 574 Gravity. In: Earth \& Space 2008: Engineering, Science, Construction, and 575 Operations in Challenging Environments, pp. 1-7.

576 Iagnemma, K., Shibly, H., Rzepniewski, A., Dubowsky, S., Territories, P., 2001. 577 Planning and control algorithms for enhanced rough-terrain rover mobility. In: The 578 6th International Symposium on Artificial Intelligence and Robotics \& Automation $579 \quad$ in Space, pp. 1-8.

580 Ishigami, G., Nagatani, K., Yoshida, K., 2006. Path following control with slip 581 compensation on loose soil for exploration rover. In: The 2006 IEEE/RSJ 582 International Conference on Intelligent Robots and Systems, pp. 5552-5557.

583 Ishigami, G., Nagatani, K., Yoshida, K., 2007. Path planning for planetary exploration 584 rovers and its evaluation based on wheel slip dynamics. In: International 585 Conference on Robotics \& Automation, pp. 2361-2366. 
586 Iwashita, K., Oda, M., 1998. Rolling resistance at contacts in simulation of shear band 587 development by DEM. Journal of engineering mechanics 124(3), 285-292.

588 Jiang, M.J., Dai, Y.S., Cui, L., Xi, B.L., 2017a. Soil-mechanics based testbed setup 589 and corresponding experimental investigation. Journal of Aerospace Engineering, $590 \quad$ accepted.

591 Jiang, M.J., Konrad, J.M., Leroueil, S., 2003. An efficient technique for generating 592 homogeneous specimens for DEM studies. Computers and Geotechnics 30(7): $593 \quad 579-597$.

594 Jiang, M.J., Leroueil, S., Konrad, J.M., 2004. Insight into shear strength functions of 595 unsaturated granulates by DEM analyses. Computers and Geotechnics 31(6), $596 \quad 473-489$.

597 Jiang, M.J., Li, L.Q., Sun, Y.G., 2012a. Properties of TJ-1 lunar soil stimulant. Journal 598 of Aerospace Engineering 25(3), 463-469.

599 Jiang, M.J, Liu, F., Shen, Z.F., Zheng, M., 2014. Distinct element simulation of 600 lugged wheel performance under extraterrestrial environmental effects. Acta $601 \quad$ Astronautica 99, 37-51.

602 Jiang, M.J., Shen, Z.F., Thornton, C., 2013. Microscopic contact model of lunar 603 regolith for high efficiency discrete element analyses. Computers and Geotechnics $60454,104-116$.

605 Jiang, M.J., Shen, Z.F., Wang, J., 2015. A novel three-dimensional contact model for 606 granulates incorporating rolling and twisting resistances. Computers \& 607 Geotechnics 65, 147-163.

608 Jiang, M.J., Sun, Y.G., Li, L.Q., Zhu, H.H., 2012b. Contact behavior of idealized 609 granules bonded in two different interparticle distances: An experimental 610 investigation. Mechanics of Materials 55, 1-15.

611 Jiang, M.J., Sun, Y.G., Xiao, Y., 2012c. An experimental investigation on the 612 mechanical behavior between cemented granules. Geotechnical Testing Journal 613 35(5), 678-690. 
614 Jiang, M.J., Xi, B.L., Arroyo, M., Rodriguez-Donod, A., 2017b. DEM simulation of

615 soil-tool interaction under extraterrestrial environmental effects. Journal of 616 Terramechanics 71, 1-13.

617 Jiang, M.J., Yu, H.S., Harris, D., 2005. A novel discrete model for granular material 618 incorporating rolling resistance. Computers and Geotechnics 32(5), 340-357.

619 Jiang, M.J., Yu, H.S., Leroueil, S., 2007. A simple and efficient approach to capturing 620 bonding effect in naturally microstructured sands by discrete element method. 621 International Journal for Numerical Methods in Engineering 69(6), 1158-1193.

622 Johnson, J. B., Kulchitsky, A. V., Duvoy, P., Iagnemma, K., Senatore, C., Arvidson, R. 623 E., et al., 2015. Discrete element method simulations of mars exploration rover 624 wheel performance. Journal of Terramechanics 62, 31-40.

625 Knuth, M. A., Johnson, J. B., Hopkins, M. A., Sullivan, R. J., Moore, J. M., 2012. 626 Discrete element modeling of a mars exploration rover wheel in granular material. 627 Journal of Terramechanics 49(1), 27-36.

628 Kobayashi, T., Fujiwara, Y., Yamakawa, J., Yasufuku, N., Omine, K., 2010. Mobility 629 performance of a rigid wheel in low gravity environments. Journal of 630 Terramechanics 47(4), 261-274.

631 Kobayashi, T., Ochiai, H., Suyama, Y., Aoki, S., Yasufuku, N., Omine, K., 2009. 632 Bearing capacity of shallow foundations in a low gravity environment. Soils and 633 foundations 49(1), 115-134.

634 Kulchitsky, A. V., Johnson, J. B., Reeves, D. M., 2016. Resistance forces during 635 boulder extraction from an asteroid. Acta Astronautica, 127, 424-437.

636 Lade, P. V., Yamamuro, J. A., Bopp, P. A., 1997. Influence of time effects on 637 instability of granular materials. Computers and Geotechnics, 20(3-4), 179-193.

638 Li, W., Huang, Y., Cui, Y., Dong, S., Wang, J., 2010. Trafficability analysis of lunar 639 mare terrain by means of the discrete element method for wheeled rover 640 locomotion. Journal of Terramechanics 47(3), 161-172.

641 Luding, S., 2008. Cohesive, frictional powders: contact models for tension. Granular 
matter 10(4), 235.

643 Medina, C., Zeghal, M., 2006. Micromechanical Modeling of the Interaction of a 644 Soil-Rigid Wheel System. In: Earth \& Space 2006: Engineering, Construction, and 645 Operations in Challenging Environment, pp. 1-8.

646 Mitchell, J.K., Bromwell, L.G., Carrier, W.D., Costes, N.C., Scott, R.F., 1972. Soil

647 mechanical properties at the Apollo 14 site. Journal of Geophysical Research $648 \quad 77(29), 5641-5664$.

649 Modenese, C., Utili, S., Houlsby, G.T., 2012. DEM modelling of elastic adhesive 650 particles with application to lunar soil. In: Earth \& Space 2012: Engineering, 651 Science, Construction, and Operations in Challenging Environment, pp. 45-54.

652 Modenese, C., Utili, S., Houlsby, G.T., 2012. A study of the influence of surface 653 energy on the mechanical properties of lunar soil using dem, in: Discrete Element 654 Modelling of Particulate Media,The Royal Society of Chemistry, 2012, pp. 69-75, 655 Nakashima, H., Aoki, S., Kanamori, H., Oida, A., 2006. Concept of Virtual Soil Bin 656 by DEM for Lunar Locomotion Studies. In: Earth \& Space 2006: Engineering, 657 Construction, and Operations in Challenging Environment, pp. 1-8.

658 Nakashima, H., Fujii, H., Oida, A., Momozu, M., Kanamori, H., Aoki, S., Yokoyama, 659 T., Shimizu, H., Miyasaka, J., Ohdoi, K., 2010. Discrete element method analysis 660 of single wheel performance for a small lunar rover on sloped terrain. Journal of 661 Terramechanics 47(5), 307-321.

662 Obermayr, M., Dressler, K., Vrettos, C., Eberhard, P., 2013. A bonded-particle model 663 for cemented sand. Computers and Geotechnics 49, 299-313.

664 Patel, N., Ellery, A., Allouis, E., Sweeting, M., Richter, L., 2004. Rover mobility 665 performance evaluation tool (RMPET): a systematic tool for rover chassis 666 evaluation via application of Bekker theory. In: The 8th ESA Workshop on 667 Advanced Space Technologies for Robotics and Automation, pp. 1-8.

668 Potyondy, D.O., Cundall, P.A., 2004. A bonded-particle model for rock. International 669 journal of rock mechanics and mining sciences 41(8), 1329-1364. 
670 Senatore C., Wulfmeier M., Vlahinic I., Andrade J., Iagnemma K., 2013. Design

671 and implementation of a particle of image velocimetry method for analysis of

672 running gear-soil interaction. Journal of Terramechanics 50, 311-326.

673 Shikanai, T., Hashiguchi, K., Nohse, Y., Ueno, M. and Okayasu, T., 2000. Precise

674 measurement of soil deformation and fluctuation in drawbar pull for steel and

675 rubber-coated rigid wheels. Journal of Terramechanics 37, 21-39.

676 Sutoh, M., Yusa, J., Ito, T., Nagatani, K., Yoshida, K., 2012. Traveling performance

677 evaluation of planetary rovers on loose soil. Journal of Field Robotics 29(4), $678 \quad 648-662$.

679 Tordesillas, A., Walsh, D.S., 2002. Incorporating rolling resistance and contact 680 anisotropy in micromechanical models of granular media. Powder Technology $681 \quad 124(1), 106-111$.

682 Wang, X.X., 2012. Calibration chamber cone penetration tests on TJ-1 lunar soil 683 simulant and DEM numerical analysis of CPT on lunar soil (Master Thesis), Tongji 684 University, China. (in Chinese).

685 Wong, J.Y., 2012. Predicting the performances of rigid rover wheels on extraterrestrial 686 surfaces based on test results obtained on earth. Journal of Terramechanics 49(1), 687 49-61.

688

689 